\title{
Explore of Modern Family in the Waste Disposal Methods
}

\author{
Caifeng Chen \\ College of Mechanical Engineering, University of Shanghai for Science and Technology \\ 516 Jun Gong Road, Shanghai 200093, China \\ Tel: 86-21-5527-0638 E-mail: chencaifeng@usst.edu.cn \\ Xinhua Wang \\ College of Mechanical Engineering, University of Shanghai for Science and Technology \\ 516 Jun Gong Road, Shanghai 200093, China \\ Tel: 86-21-5527-3617 E-mail: wangxinhua@hotmail.com
}

\begin{abstract}
In this paper we proposed the development scheme of the modern family's kitchen waste processing machine combined with automatical crush, dewatering, drying, granulation and sterilization, and discussed the proper way of process to reduce, recycle and reuse the modern family's kitchen waste by analyzing the production condition, present process and processing technology.
\end{abstract}

Keywords: Kitchen waste, Crush, Dewater, Drying

\section{Introduction}

Shanghai, for example, the daily waste in shanghai is more than million tons, and increases by $10 \%$ according to statistics. The cook waste accounts for $40 \%$ of the total. There are about 7 million families in Shanghai, every family produces the cook waste more about $1.5 \sim 2 \mathrm{~kg}$, the daily waste in shanghai is more about 1200 tons accordingly, the family is the main source of the city's waste. For the big population, fast urbanization and high comprehensive handling charges in China, it is imperative to control the cook waste from the source and realize "reduce no harm resource".

The kitchen waste is produced easily rotten wastes by family. The common components are food remainder, waste oils and greases, dishes vegetable leaves and leftovers for fruit peel organic waste etc. The Characteristics of the cook waste is oily and wet, rotten easily, nutrient, and with existence of the virus and pathogens microorganisms, it is difficult to the collect, transport and handle. Some waste disposal facilities have been used at home and family, they are mainly mechanical facilities (principle of rapid speed and crystal), heat facilities (Heat carbonization principle) and biological facilities (Bacteria groom principle) etc. The equipment exists some problem or inconvenience in use so that they might be promoted difficultly.

(1) Mechanical equipment: This is suspended at the bottom of the kitchen sink, and the cook rubbish goes from the bottom of the kitchen sink into the downfall container, finally drain away the downfall rubbish from the water pipe, but this rubbish is easily clogged in the water pipe, and it is easy to gather dirt, to distribute smells and breed insects in the inside wall of downfall warehouse. The sink is easier to shape and it may lead to leakage and blow out the electrical motor.

(2) Heating equipment: A processing time is very long, and usually continues two to four hours. It also consumes large electricity and stores difficultly; it is going to wash by hand the oil of the cook rubbish and then put the waste broken or filter water by hand before drying, and finally dries it. It is inconvenient and even has some cumbersome.

(3) Biological device the user needs displace the bacteria regularly, it not only displaces difficultly but also biological bacteria easily make the user fear. Its price is also too high to go into average homes of the city.

therefore, the article proposes the scheme of the automation process equipment for the cook rubbish of modern urban families Integrating with downfall, dehydrated, drying and making particles, disinfection and storage (abbreviation for the kitchen waste processor), it can automatically process the domestic food waste safely and conveniently, and after handling they turn into dry Powder or particles and are turned into raw materials of organic and feed to realize the purpose of "reduce no harm resource" for cook rubbish.

\section{The Structure design of the cook rubbish processor}

\subsection{Design requires}

Based on the situation of about $1.5 \sim 2 \mathrm{~kg}$ made by the daily waste in shanghai and the requirements for high efficiency and low energy consumption, the technological requirements for the remainder processor is that the processing waste for a time is $1.0 \mathrm{~kg}$, the smash cavity is $2.5 \mathrm{~L}$, the volume of microwave drying Cavity is $3.5 \mathrm{~L}$, the volume of the storage for drying material is $5 \mathrm{~L}$, for 5 . The drinking water for a time is Less than or equals $7 \mathrm{~L}$, the electricity consumption for a time is Less than or equals 0.5 degrees, and a single processing time is Less 
than or equals 45 minutes.

\subsection{Overall structure design}

The waste processor consists of three parts and four devices. The three parts are smash mechanism, dewater and mechanism for delivering material and mechanism for drying up and making particles. The four devices are the washing and reverse rushing device with the high pressure mist and water, the sensing device of temperature and humidity, device for drying and disinfecting with microwave and purification device for waste fumes.

\subsection{Smash mechanism}

The highest hardness for the remainder is poultry bones and teeth; we usually impact them with the hammer for pieces fully, as shown in figure 2. It is equipped with fix teeth and crystal knife plate with multiple functions in the downfall cavity and it is equipped with two moving hammer on the crystal knife plate. The remainders go to the downfall room from the entrance; the spinning knife plate driven by the motor turns the two moving hammer and fix teeth into spinning knife groups. The rubbish is broken into pieces by the joint action of compressing, impacting, grinding, splitting, and cutting and then drains by the crystal force of the teeth and goes into dewater mechanism from channels.

\subsubsection{Structural design of the Centrifugal plate}

The centrifugal knife plate is the key components of the smash mechanism as shown in figure 2. The requirements for it are resisting to corrosion and high hardness, strength and stiffness, so we choose Martensitic stainless steel material, the diameter of the centrifugal knife plate is $120 \mathrm{~mm}$ referring to the structural dimension of the kitchen sink on test.

\subsubsection{Force analysis for the centrifugal knife plate and the determination of power}

Based on the actual applied load of the centrifugal knife plate, we idealize the knife plate to the cardboard rotating by the central axis. There are fluid resistance, resistance when breaking the waste (cut, tear and wear) resistance and fluid pressure.

(1) Fluid resistance $F_{1}$ : According to the following formula, calculate the moment-of-fluid resistance on the centrifugal knife plate:

$$
M=3.87 R^{3}+\frac{1}{2} \rho \mu \sqrt{\left(\gamma / u_{1} R\right)^{\frac{1}{2}}}
$$

In the formula above, viscosity $\gamma=0.01 \mathrm{~cm} / \mathrm{s}$, fluid density $\rho=1.0 \times 103 \mathrm{~kg} / \mathrm{m} 3$, Poisson ratio $\mu=0.5 \mathrm{~N} / \mathrm{m}$, radius of the centrifugal knife plate $\mathrm{R}=0.06 \mathrm{~m}$, line speed $\mathrm{u} 1=\omega \mathrm{R}$, angular velocity angel Speed $\omega=2 \pi \mathrm{n} / 60 \mathrm{rad} / \mathrm{s}$, rotating speed of the Crushing motor $n=2800 \mathrm{r} / \mathrm{min}$, Calculation: the moment-of-fluid resistance $\mathrm{M}=0.12 \mathrm{~N}$.m, so fluid resistance $\mathrm{F} 1=2 \mathrm{~N}$.

(2) Resistance from the waste $\mathrm{F}_{2}$ : Through testing on the common poultry bones and scraps from the waste, work out the biggest resistance by testing $\mathrm{F}_{2}=11.5 \mathrm{~N}$.

(3) Fluid pressure $\mathrm{F}_{3}$ : In formula, the fluid depth $\mathrm{h}$ is $150 \mathrm{~mm}$, fluid pressure $\mathrm{q}$ can be calculated, $\mathrm{q}=\rho \mathrm{gh}$ $=1.0 \times 103 \times 9.8 \times 0.15=1470 \mathrm{~N} / \mathrm{m}^{2}$, so fluid pressure $\mathrm{F}_{3}=\mathrm{qA}=\mathrm{q} \pi \mathrm{R}^{2}=1470 \times \pi \times 0.06^{2}=16.6 \mathrm{~N}$.

(4) The overall resistance to overcome $F: F=F_{1}+F_{2}+F_{3}=2+11.5+16.6=30.1 \mathrm{~N}$

(5) Determining power of downfall electrical motor $P_{w}$

When the downfall knife plate working, the resistance to overcome is $\mathrm{F}$, rotating speed of the centrifugal knife plate $\mathrm{n}=2800 \mathrm{r} / \mathrm{min}$, so line speed $\mathrm{V}=15.5 \mathrm{~m} / \mathrm{s}$, so the working power of knife plate $\mathrm{P}_{\mathrm{w}}=\mathrm{FV}=468 \mathrm{~W}$.

\subsection{Dehydration and delivering material mechanism}

The mature method for processing kitchen waste utilizes comprehensively comminuting and suppressing and dehydrating and dry technology. The machine chooses a spiral squeezing mechanism to divide solid and liquid for squeezing continuously, the design adopts a small power electrical motor ( $25 \mathrm{w}$ motor of dehydration), and it rotates at a low speed and has low energy consumption and noise reduction.

The spiral squeezing mechanism is shown as in figure 3, it consists of the cylinder shell and rotating shaft. It is fitted with a filter net in the cylinder shell. The spiral blade pushes the material from the feed holes to the export. Since the entrance volume of spiral shaft and shell is reduced gradually, the materials are squeezed, the liquid is drained through the filter hole, and then the solid materials go to the drying mechanism from the hole of draining material.

\subsection{Dry, mixture and particle made mechanism}

The volume of the mechanism is so big that it is placed at the bottom of the machine to ensure stability of the whole machine and., as shown in figure 1 , the structure of the mixture axis is shown in figure 3 , the blade is welded in the stir bar, the process is better to mix fully in drying up under high temperature and materials are heated uniformly. The heat way by microwave can ensure the disinfection effectiveness.

\section{Process automatically in the cook waste}

In the test, after placing the remainder in the downfall cavity, press down "run" key, the device will start 
automatically and end after 45 minutes. The whole process as the following:

1) Empty food waste into the smash cavity;

2) Wash away food grease with high pressure misty water;

3) Drain grease to drainage;

4) Grid food waste by injecting water;

5) Deliver particles and dewater by centrifugal force;

5) Drain waste gas to drainage;

7) Dry and grid materials and deliver automatically;

8) Wash out cavity and drain water;

9) Store materials to storage box;

\section{Conclusion}

The cost of the development programme for the kitchen waste processor in the article is about half of that of biological processor, and convenient in using and compact in structure. The drying grainy can be turned into the raw materials for organic fertilizer and feed, thus reduce environment pollution and threaten of the kitchen waste and produce good social significance.

\section{Acknowledgment}

This work is supported by Shanghai Leading Academic Discipline Project, Project Number: J50503.

\section{References}

Zhang, Xianhui, Zhang, Bo \& Yi, Xiaohong. (2005). Discussion on Method of food wastes Disposal. Environmental Science and Mangement. 2006(2) 141 142.

Wang, Xianhui, Li,Guangwei, Meng, Hong \& Zhao Guojue. (2005). Discussion on Treatment Status of Food Residue. Enviornmental Sanitation Engineering.2005(4). 41 43.

Zeng, Qigao. (2008). Design of Control System for Remainder Processor Based on the PLC. Electrical and mechanical engineering technology. 2008(12). 28 29.

Zhang, Xinhui, Zhang, bo \& Yi, xiaohang. (2006). Explore for handling the cook rubbish. Environmental science and manage. 2006 (2).

Song sixin. (2006). Design and study for moving components of smash processor based on adams and ansys. Beijing university of chemical journal. 2006 (5).

Xin, Xiaomu. (2008). Cleaner Production and Analysis of Urban Domestic Waste treatmenys. Journal of meteorology and environment. 2008(4). 50 53.

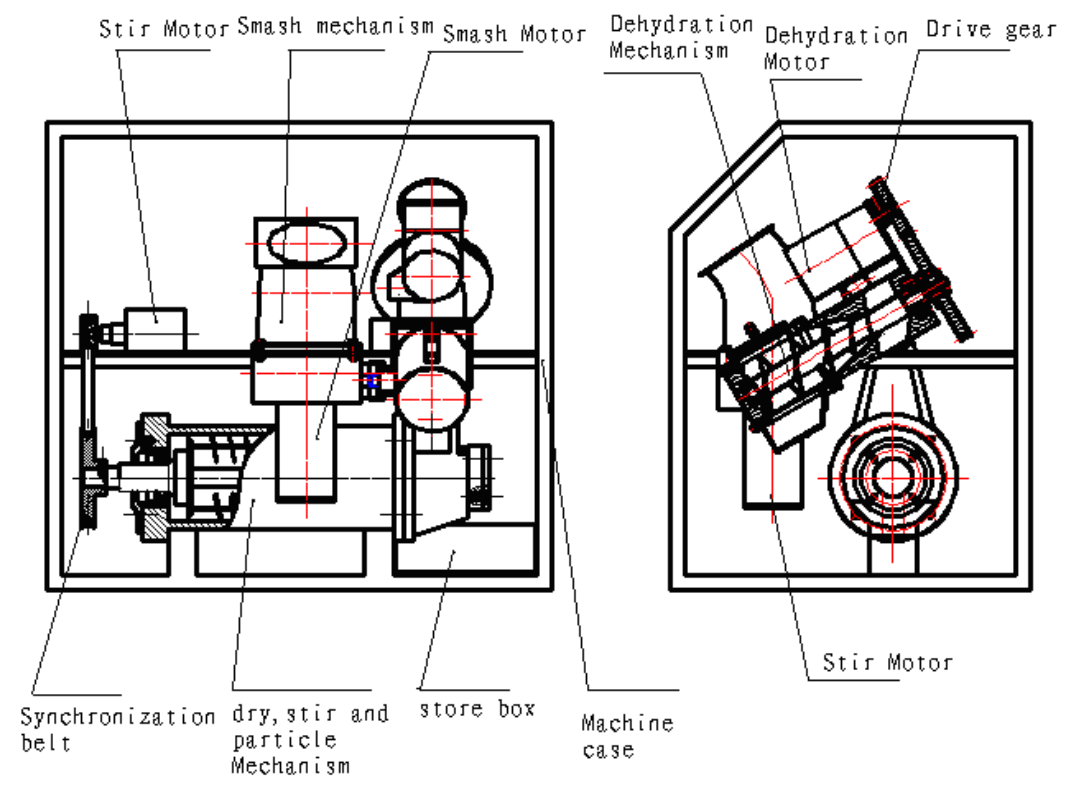

Figure 1. Diagram of internal structure layout 


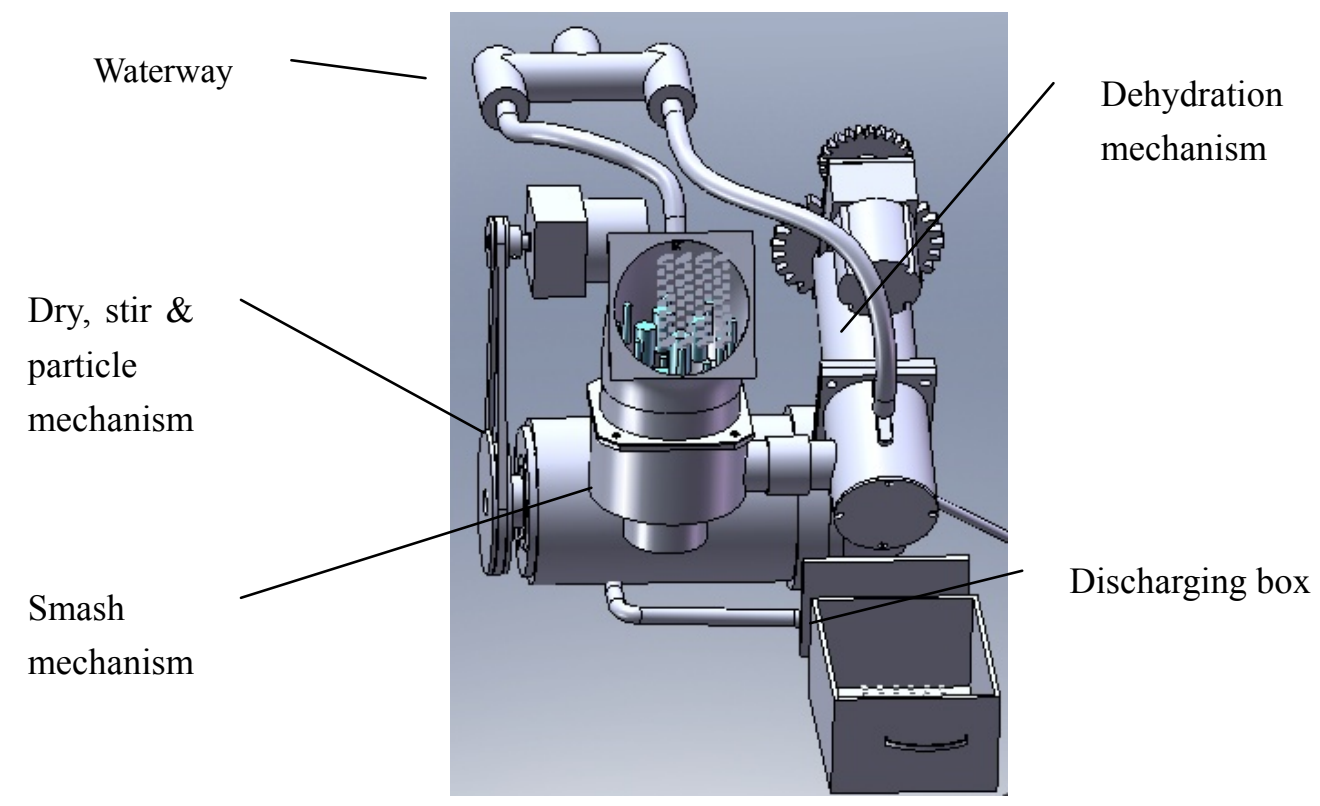

Figure 2. 3d graphics of internal structure layout

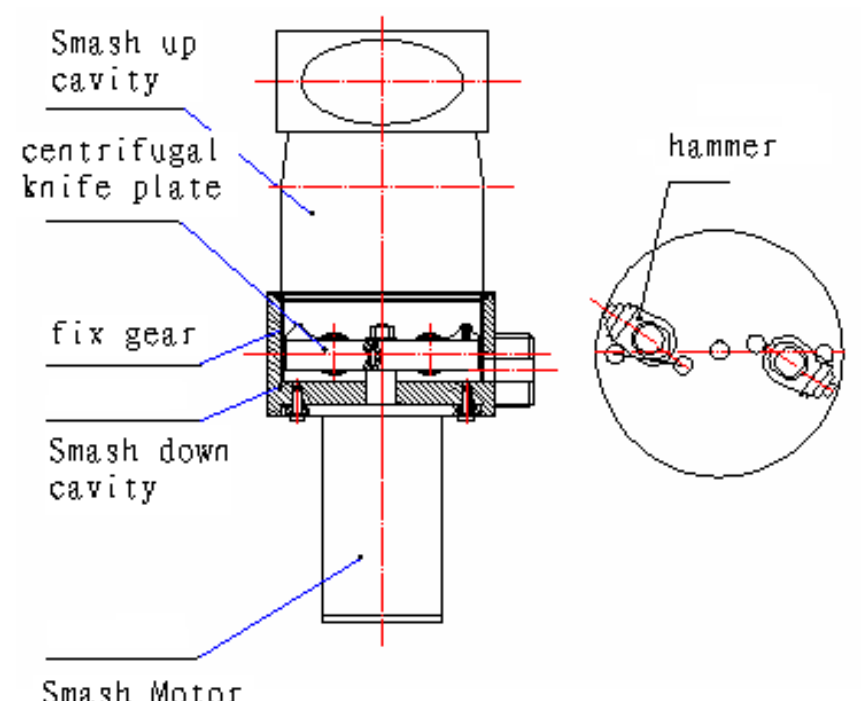

Smash Motor

Figure 3. Diagram of the comminuting mechanism

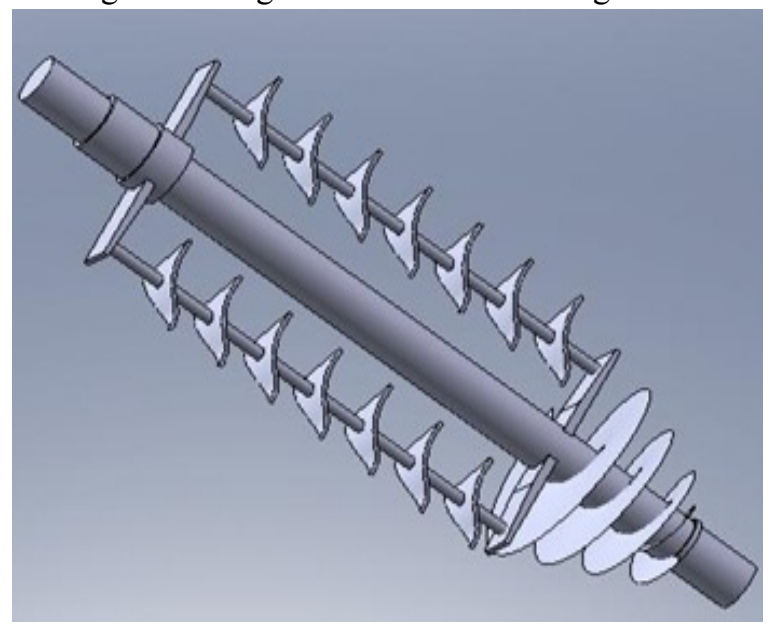

Figure 4. Diagram of the stir shaft 